\title{
Tumor treating fields is a newly epoch making therapeutic method for malignant brain tumors
}

\begin{abstract}
Glioblastoma (GBM) is a malignant brain tumor and extremely difficult to treat with no specific curative treatment. Tumor treating fields (TTFields/TTF) are a new cancer treatment modality for patients with GBM, which uses alternating electrical fields. This review introduces the principle of TTF and reports on recent GBM treatments and prospects.
\end{abstract}

Keywords: tumor treating fields, glioblastoma, aalternating electric fields

\author{
Volume I Issue I - 2017 \\ Sadahiro Kaneko,' Sadao Kaneko \\ 'Department of Neurosurgery, Tomakomai City Hospital, Japan \\ ${ }^{2}$ Department of Neurosurgery, Kashiwaba Neurosurgical \\ Hospital, Japan
}

\begin{abstract}
Correspondence: Sadao Kaneko, Department of Neurosurgery, Kashiwaba Neurosurgical Hospital, 7-20, I5-chome, I-jyou, Tsukisamuhigashi, Toyohira-ku, Sapporo, Hokkaido 062-58I3, Japan, Tel +8I-I I-85I-2333,

Email srkaneko@seagreen.ocn.ne.jp
\end{abstract}

Received: June 27, 2017 | Published: July 07, 2017

\author{
Abbreviations: GBM, glioblastoma; TTFields/TTF, tumor \\ treating fields
}

\section{Introduction}

Glioblastoma (GBM) possesses a lethal prognosis. Despite surgery, radiotherapy, and chemotherapy, the median survival time for a patient with GBM is only 15.0 months. TTF have a very different mechanism from conventional treatment methods in which malignant tumor cells placed in a specific electric field environment are broken down and lead to apoptosis. Several multicenter studies to treat GBM using TTF have been performed so far and have shown superior results.

\section{Discussion}

\section{Basics and theory of TTF}

To date, many studies have shown the influence of the electric field on the body. Kirson et al. ${ }^{1}$ explained the basic principle of TTF in $2004 .{ }^{1}$ TTF create an alternating electric field in the brain that inhibits cell division, thereby causing cells to trigger apoptosis. TTF are thought to be effective in two different phases during cell division. First, when microtubules are polymerized and mitotic spindles are formed (from the early to middle stages of mitosis), an electric field inhibits polymerization and cell division, leading to apoptosis. Second, the cells take an hourglass-like form in the late stage of mitosis and during cytokinesis, and a slight electric field change occurs in the cells. At this time, when the electric field is applied, the component is pushed out toward the constricted part of the form, destroying the structure of the cell and leading to apoptosis. TTF utilize the effect obtained by providing an electric field to the cells at the time of cytokinesis from mitosis in a very brief period during cell division and inhibits cell division. As a matter of course, this therapy has no effect on cells that are not dividing. Furthermore, it has the highest therapeutic effect when the axes of division and electric field are parallel, but when it is at a right angle, the effect becomes the lowest. Therefore, it does not mean that all cells are affected by the electric field and cause apoptosis.

\section{Clinical trial for GBM using TTF}

Stupp et al. $^{2}$ reported EF-11 in $2012^{2}$ as the first multicenter randomized clinical trial conducted using TTF for GBM. Patients with recurrent GBM were randomized to receive a treatment with TTF alone $(n=120)$ or chemotherapy $(n=117)$. The median overall survival (OS) after randomization was 6.6 versus 6.0 months. The median progression free survival (PFS) rate was 2.2 and 2.0months. No significant difference was observed among them. However, significantly fewer systematic side effects were observed with TTF alone. Gastrointestinal disorders, blood system disorders, and infections, which are typical adverse events of chemotherapy, were significant low in the TTF alone. Contact dermatitis of the scalp, which is an adverse event related to TTF, was found in $16 \%$ of patients and could be treated only by a steroid ointment application. This result showed that toxicity and quality of life clearly favored TTF.

Stupp et al. ${ }^{3}$ reported the EF-14 trial as a phase 3 randomized clinical trial of TTF for newly diagnosed GBM in 2015. ${ }^{3}$ After completion of chemoradiotherapy, patients with newly diagnosed GBM were randomized for treatment with TTF plus temozolomide maintenance therapy $(\mathrm{n}=466)$ or temozolomide maintenance therapy alone $(n=229)$. The median OS was 19.6 months in the TTF plus temozolomide group and 16.6 months in the temozolomide alone group, and the median PFS was 7.1 and 4.0months, respectively. These result showed that TTF significantly prolongs PFS and OS in patients with newly diagnosed GBM.

\section{Prospects}

It is expected that TTF become a new cancer treatment modality for patients with GBM. Because of its mechanism of action, TTF therapy alone only effects cells in the division phase, but it has been reported that enhance a cell proliferation inhibitory effect in combination with chemotherapy and affect cell self-repair function. ${ }^{4,5}$ Currently, clinical trials are being planned for GBM as well as for lung, pancreatic, breast, and ovarian cancers. 


\section{Conclusion}

In recent years, TTF have gained attention as a new treatment for GBM. Many multicenter clinical trials have shown that TTF prolong the prognosis without weakening Activity of Daily Living.

\section{Conflict of interest}

The authors declare that there are no conflicts of interest regarding to the publication of this paper.

\section{References}

1. Kirson ED, Gurvich Z, Schneiderman R, et al. Disruption of cancer cell replication by alternating electric fields. Cancer Res. 2004;64(9):3288-3295.
2. Stupp R, Mason WP, van den Bent MJ, et al. Radiotherapy plus concomitant and adjuvant temozolomide for glioblastoma. $N$ Engl J Med. 2005;352(10):987-996.

3. Stupp R, Taillibert S, Kanner AA, et al. Maintenance Therapy With Tumor-Treating Fields Plus Temozolomide vs Temozolomide Alone for Glioblastoma: A Randomized Clinical Trial. Jama. 2015;314(23):2535-2543.

4. Kirson ED, Schneiderman RS, Dbalý V, et al. Chemotherapeutic treatment efficacy and sensitivity are increased by adjuvant alternating electric fields (TTFields). BMC Med Phys. 2009;9(1):1756-6649.

5. Schneiderman RS, Shmueli E, Kirson ED, et al. TTFields alone and in combination with chemotherapeutic agents effectively reduce the viability of MDR cell sub-lines that over-express $\mathrm{ABC}$ transporters. $B M C$ Cancer. 2010;10(229):1471-2407. 